\title{
The characterization of prokaryote strains for taxonomic purposes
}

The number of proposals of novel species and novel genera published in the IJSEM is still increasing and it can be expected that this will be a continuing trend. This is a reflection of the tremendous diversity of prokaryotic organisms that can be detected and isolated from all kinds of environments that fulfil the minimum prerequisites for life and survival. A comprehensive characterization of a strain is a key element in prokaryotic systematics. Over the past decades, the methods for characterization of bacterial and archaeal strains have been considerably expanded.

Very early investigations of prokaryotes were dominated by a strong emphasis on morphology and physiology. Beginning in the 1950s, new developments in the application of numerical phenetics, numerical taxonomy, chemosystematics and finally molecular systematics have revolutionized the classification of prokaryotes. In particular, the application $16 \mathrm{~S}$ rRNA gene sequencing has provided a basis for studies of the natural relationships among prokaryotic organisms. However, although $16 \mathrm{~S}$ rRNA gene sequence analyses have provided a framework for prokaryotic classification, the current classification system based on this molecule alone has not yet solved the taxonomic problems among and within numerous genera. As a consequence, methods considered as being 'traditional' remain key elements in determining whether a strain belongs to a known taxon or constitutes a novel one.

In many laboratories, the first step to characterize a bacterial or archaeal strain or a set of strains is the 16S rRNA gene sequencing approach. However, in the case of a strain or set of strains presumptively representing novel taxa (often indicated by low 16S rRNA gene sequence similarities to sequences of existing taxa), they should be characterized as comprehensively as possible. In order to give some 'guidelines' for this complex procedure, the 'Notes on the characterization of prokaryotic strains for taxonomic purposes' (Tindall et al., 2010) have been devised. This taxonomic note deals with current methodologies and outlines how these methods should be used in an integrated manner. It is not intended to guide the reader as to how the results should be interpreted, although some information is given as to when the value of the dataset may be helpful. The article covers genetic (including protein sequence-based methods) and phenotypic methods. The latter in particular are exemplified in great detail as it seems that the knowledge of and experience with these 'traditional' methods is fast disappearing.

Although there is currently a strong movement towards a genomic oriented taxonomy, phenotypic characterization remains an indispensable element of prokaryotic taxonomy. The increasing number of sequenced genomes from a diverse range of prokaryotes is providing an interesting addition to the methods that are now considered to be 'traditional'. At the same time, some of the methods listed in the 'guidelines' provide a more detailed insight into the structure of the cells and can reveal where information is currently scant or lacking at the genomic level. It should be emphasized that only the interplay between genetic and phenotypic datasets can provide a sound basis for appreciating and describing the diversity of prokaryotes. Such a combined approach has the potential to become the foundation of a more stable, in-depth taxonomy of the prokaryotes. It is hoped that this set of guidelines will serve taxonomists all over the world and will also have implications for other areas of microbial research.

\section{Peter Kämpfer}

Institut für Angewandte Microbiologie, Justus-Liebig-Universität Giessen, Heinrich-Buff-Ring 26-32 (IFZ), D-35392, Giessen, Germany

Correspondence: Peter Kämpfer (peter.kaempfer@agrar.uni-giessen.de)

Tindall, B. J., Rosselló-Móra, R., Busse, H.-J., Ludwig, W. \& Kämpfer, P. (2010). Notes on the characterization of prokaryote strains for taxonomic purposes. Int J Syst Evol Microbiol 60, 249-266. 